\title{
Laparoscopic Management of a 13 Week Interstitial Ectopic Pregnancy
}

\author{
Ariel K. Dubin, MD, Eve F. Zaritsky, MD, Miya P. Yamamoto, MD \\ Department of Obstetrics and Gynecology, Kaiser Permanente Santa Clara, Santa Clara, California (Dr Dubin); \\ Department of Obstetrics and Gynecology, Kaiser Permanente Oakland, Oakland, California (Dr Zaritsky); Department \\ of Obstetrics and Gynecology, Kaiser Permanente San Leandro, San Leandro, California (Dr Yamamoto).
}

\begin{abstract}
Introduction: Interstitial pregnancies account for approximately $2 \%$ to $4 \%$ of all ectopic pregnancies; however, without timely diagnosis and treatment, they are associated with a high rate of morbidity and mortality. The traditional surgical approach has been laparotomy with cornual wedge resection or even hysterectomy. With advances in laparoscopic surgery, new surgical techniques have been reported: cornuostomy incision with removal of pregnancy contents, cornual wedge resection, and combined laparoscopic and hysteroscopic suction removal. However, these laparoscopic techniques have been described mostly in first-trimester interstitial pregnancies with smaller mass sizes.

Case Description: We report a case of a successfully treated interstitial pregnancy of a 35-year-old woman in her second trimester with a mass diameter of $8 \mathrm{~cm}$ via laparoscopic cornual wedge resection. The surgery had minimal blood loss, aided by myometrial injection of dilute vasopressin, systematic electrocautery ligation, and continuous barbed suture closure of the remaining myometrium and serosa. The patient had an uneventful postoperative course and has recovered fully.

Discussion: With advances in laparoscopic techniques, laparoscopy has become a viable surgical treatment of interstitial pregnancy. However, few case descriptions of successful laparoscopic management of pregnancies that have progressed into the second trimester exist. This is due to several factors: the rarity of these pregnancies to progress past the first trimester without uterine rupture, the size of second-trimester pregnancies, and the technical difficulty encountered with such large resections. In our case report, we share our successful experience of laparoscopic management of an 8-cm-diameter interstitial pregnancy at 13 weeks' gestation.
\end{abstract}

Key Words: Ectopic pregnancy, Interstitial pregnancy, Laparoscopy, Laparoscopic cornual wedge resection.

Citation Dubin AK, Zaritsky EF, Yamamoto MP. Laparoscopic management of a 13 week interstitial ectopic pregnancy. CRSLS e2014.00226. DOI 10.4293/JSLS.2014.00226.

Copyright (C) 2014 SLS This is an open-access article distributed under the terms of the Creative Commons Attribution-Noncommercial-ShareAlike 3.0 Unported license, which permits unrestricted noncommercial use, distribution, and reproduction in any medium, provided the original author and source are credited.

Address correspondence to: Ariel K. Dubin, MD, Kaiser Permanente Santa Clara, Department of Obstetrics and Gynecology, 710 Lawrence Expressway, Santa Clara, CA 95051. Tel: 408-851-3842, Fax: 408-851-3839, E-mail: ariel.k.dubin@kp.org.

\section{INTRODUCTION}

Extrauterine pregnancies complicate approximately $1 \%$ to $2 \%$ of all pregnancies. Approximately $95 \%$ of extrauterine pregnancies are located in the fallopian tube. ${ }^{1,2}$ However, it is important to remember that $5 \%$ of ectopic pregnancies occur elsewhere.

The most proximal portion of the tube, located within the myometrium, is called the interstitium. It is a tortuous segment of the tube that is $0.7 \mathrm{~mm}$ wide and 1 to 2 $\mathrm{cm}$ long. Pregnancies that implant in the interstitium are designated as interstitial ectopic pregnancies. Interstitial pregnancies are commonly but mistakenly referred to as cornual or angular. A distinction should be made, as treatment and prognosis of the 3 conditions differ significantly. A cornual pregnancy is a pregnancy that occurs in 1 of the horns of a bicornuate uterus or a rudimentary horn of a unicornuate uterus. An angular pregnancy occurs when implantation is in the lateral angles of the uterine cavity, medial to the uterotubal junction, but it is a viable pregnancy as it is covered by endometrium. ${ }^{3,4}$ 
Interstitial pregnancies account for just $2 \%$ to $4 \%$ of all ectopic pregnancies; however, the reported maternal mortality is in the range of $2 \%$ to $2.5 \%, 7$ times higher than more common tubal pregnancies. ${ }^{5}$ This high mortality rate is due in part to diagnostic difficulty, resulting in delayed diagnosis and a higher rate of uterine rupture and resultant bleeding. Differentiating an eccentrically located intrauterine pregnancy (such as an angular pregnancy) and interstitial pregnancy is difficult because both can appear intrauterine. The following criteria on ultrasound may aid in the diagnosis of an interstitial pregnancy: (1) a gestational sac $<1 \mathrm{~cm}$ from the serosa, (2) asymmetric thinning of the myometrium, (3) absence of endometrium, and (4) the "interstitial line sign," an echogenic line that extends into the upper regions of the uterine horn and borders the margin of the intramural gestation sac, possibly representing the interstitial tube or endometrium. ${ }^{6-8}$

Rupture of the uterus occurs in up to $20 \%$ of the cases that progress past the first trimester. ${ }^{5}$ This creates a dangerous and difficult challenge to providers: it is difficult to diagnose these pregnancies, especially early in gestation, but the consequences of a missed diagnosis can be dire.

Ipsilateral salpingectomy and assisted reproductive technologies such as in vitro fertilization are predisposing factors specific to interstitial ectopic pregnancies. In addition, general risk factors for ectopic pregnancy such as history of pelvic inflammatory disease, previous pelvic surgery, uterine anomalies, and previous ectopic pregnancy place patients at increased risk. ${ }^{8}$

Although previously believed to rupture later in gestation than tubal pregnancies because of the thicker uterine wall, interstitial pregnancies have been shown in studies to rupture relatively early in gestation. ${ }^{8}$

There are a variety of clinical decision-making algorisms in the literature to guide the management of eccentrically located intrauterine pregnancies. Treatment then depends on the presenting findings, including gestational age, $\beta$ human chorionic gonadotropin (BHCG) levels, and patient symptoms. 9 The key factor is the location or suspected location of the pregnancy and the size of the pregnancy. In a confirmed interstitial pregnancy, the traditional surgical treatment has been hysterectomy or cornual wedge resection via laparotomy, primarily because of the high morbidity associated with myometrial surgery due to increased risk for bleeding and the technically difficult surgical approach. ${ }^{4}$ However, a laparotomy can lead to a multiday hospital stay, increased postoperative pain, increased wound complications, a prolonged recovery, lost wages, and high health care costs. ${ }^{10}$ More con- servative treatments and minimally invasive surgical techniques have been developed to avoid these negative outcomes. Laparoscopic and hysteroscopic surgery, methotrexate administration, and pregnancy termination by potassium chloride injection have been described. An excellent review by Moawad et $\mathrm{al}^{4}$ describes these techniques. Systemic methotrexate either in a single- or multiple-dose regimen has been used for successful treatment of early first trimester interstitial pregnancies. Local injection of methotrexate or potassium chloride has been described in conjunction with surgical removal, but these approaches are less commonly reported.

With the advent of laparoscopic surgery and subsequent rapid advances in operative techniques, laparoscopic approaches have been used to successfully treat more complex gynecologic conditions, including interstitial pregnancies. Level I evidence from a Cochrane review showed that when performed by trained laparoscopic surgeons, the laparoscopic approach to major surgical procedures, including hysterectomy, results in significantly shorter hospital stays, faster recoveries, and reduced blood loss and wound complications. ${ }^{10}$ Several laparoscopic techniques have been described, including cornuostomy incision with removal of the gestational sac and pregnancy contents, cornual wedge resection, and combined laparoscopic and hysteroscopic suction removal. ${ }^{3,11-17}$ These techniques are aided by several methods to minimize blood loss during surgery: local injection of dilute vasopressin to constrict local blood vessels, electrocoagulation at the area of dissection, occlusion of the ascending branch of the uterine artery via suture ligation or electrocautery, and multilayered closure of the defect. ${ }^{4}$

Although laparoscopic surgery improves many markers of morbidity and mortality as noted above, a serious complication, uterine rupture, can occur in a subsequent pregnancy. This complication has been studied after myomectomy procedures and has been proposed to follow laparoscopic removal of interstitial pregnancies because of the similarities in operative technique. Parker et al ${ }^{18}$ reviewed 19 cases of uterine rupture after laparoscopic myomectomy and identified several factors that may bring about this rare but catastrophic complication in a subsequent pregnancy. They suggested that, in comparison with techniques used in abdominal myomectomy, the reliance on electrocautery for hemostasis may play a role in poor myometrial healing and weaker scar formation. Parker et $\mathrm{al}^{18}$ and Cobellis et $\mathrm{al}^{19}$ also suggest that closing the myometrial incision in several layers can improve scar thickness and therefore strength. Two studies of abdominal myomectomies suggest that at least 2-layer closure of myometrium and 
single-layer closure of the serosa are ideal for maximizing scar integrity. ${ }^{20,21}$ This issue is especially important for larger resections, as we describe in our case report.

Many of the previously reported cases have described successful laparoscopic treatment for early interstitial pregnancies (first trimester) on average between 8 and 9 weeks. ${ }^{3}$ Here, we report the case of a successfully treated second-trimester interstitial pregnancy with a mass diameter of $8 \mathrm{~cm}$ via laparoscopic cornual wedge resection with minimal blood loss aided by myometrial injection of dilute vasopressin and electrocautery aided resection.

\section{CASE DESCRIPTION}

A 35-year-old G4P1021 woman at approximately 13 weeks' gestation presented for routine nuchal translucency ultrasound. The patient presented with 10 days of vague, dull lower pelvic pain that was nonlocalized and had not required medications. Her obstetric history was significant for an ectopic pregnancy (location unknown) 14 years before presentation treated with methotrexate therapy, 1 term normal vaginal delivery 13 years before presentation, and 1 uncomplicated surgical therapeutic abortion at 8 weeks' gestation 4 years before presentation. The initial nuchal translucency ultrasound study, conducted by a perinatologist, was concerning for an eccen- trically located gestational sac without measurable myometrium at the right cornua measuring 13 weeks 0 days. The suspicious findings were confirmed with a second ultrasound examination by a radiologist, with estimated gestational age of 13 weeks 5 days (Figure 1). The size of the mass on ultrasound was $8.0 \times 6.5 \mathrm{~cm}$. The BHCG level was noted at $55,359 \mathrm{mIU} / \mathrm{mL}$.

\section{Technique Description}

The patient provided consent for a laparoscopic uterine wedge resection, possible unilateral salpingo-oophorectomy, hysterectomy, and laparotomy. Wedge resection was chosen as the operative approach to maximize the amount of pregnancy tissue that could be easily identified and removed. Because of the advanced gestational age, size of the mass, lack of circumferential surrounding myometrium, and abdominal pain, there was high concern for uterine rupture, so the decision was made to proceed immediately to the operating room for surgery. Therefore, methotrexate with serial BHCG surveillance was not considered a feasible alternative. Although successful use of intra-amniotic potassium chloride injection in the treatment of cervical ectopic pregnancies without a plan for surgical intervention has been reported, few large studies have shown the advantages of preoperative potassium

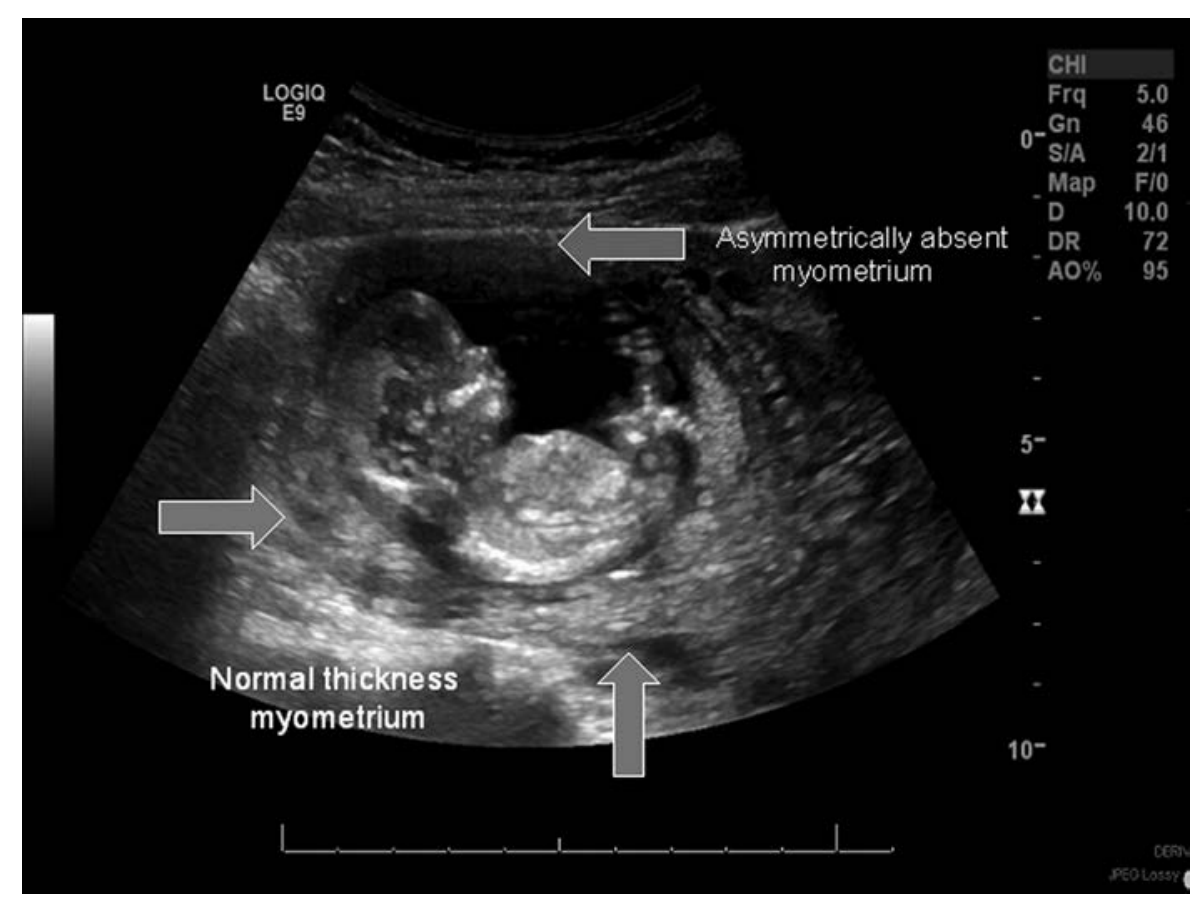

Figure 1. Transabdominal ultrasound image showing an interstitial pregnancy at 13 weeks 5 days. Arrows indicate normal endometrium on the left and absent myometrium above the pregnancy. 


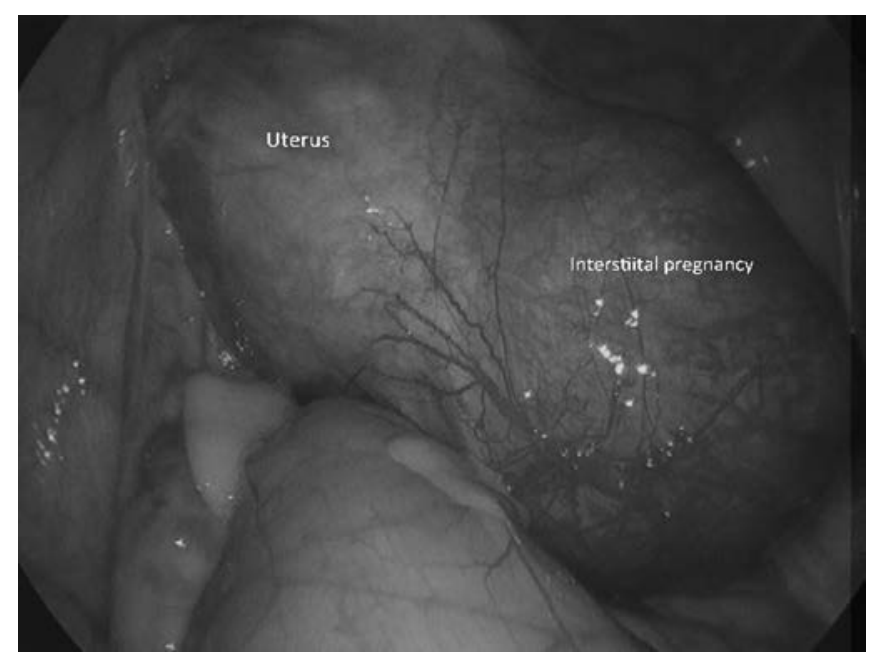

Figure 2. Laparoscopic view of the uterine fundus with an enlarged mass equal to the size of the uterus at the right cornua. Note the hypervascularity and hyperemic appearance of the serosa overlying the pregnancy.

chloride use in advanced interstitial pregnancies. Without clear benefit to potassium chloride use, such as decreased surgical blood loss, the decision was made to proceed without using this medication.

Operative findings showed the visible absence of myometrium of a 13-cm right interstitial ectopic pregnancy, with a normal right ovary (Figure 2).

Before abdominal entry, the cervical stroma was infiltrated with dilute vasopressin (approximately $20 \mathrm{~mL}$ of a dilution of $20 \mathrm{U}$ vasopressin in $100 \mathrm{~mL}$ normal saline), with the goal of minimizing blood loss by vasoconstriction. A vaginal-cervical Ahluwalia retractor-elevator (VCARE; ConMed, Utica, New York) was placed for uterine manipulation and to aid in colpotomy incision if a hysterectomy was necessary.

Three 5-mm laparoscopic ports were used to complete the following surgery: $40 \mathrm{~mL}$ of the dilute vasopressin solution was injected circumferentially into the myometrium at the base of the mass to reduce blood loss. The mass was systematically coagulated and ligated circumferentially working inward until it was removed from the uterus using the PK Gyrus (Olympus America, Center Valley, Pennsylvania) (Figures 3 and 4).

The remaining myometrial defect was reapproximated in 4 layers using a 2-0 V-Loc wound closure device (Covidien, Dublin, Ireland) with a standard laparoscopic suturing technique (Figures 5 and 6). No adhesion barrier was placed. Estimated blood loss was $50 \mathrm{~mL}$.

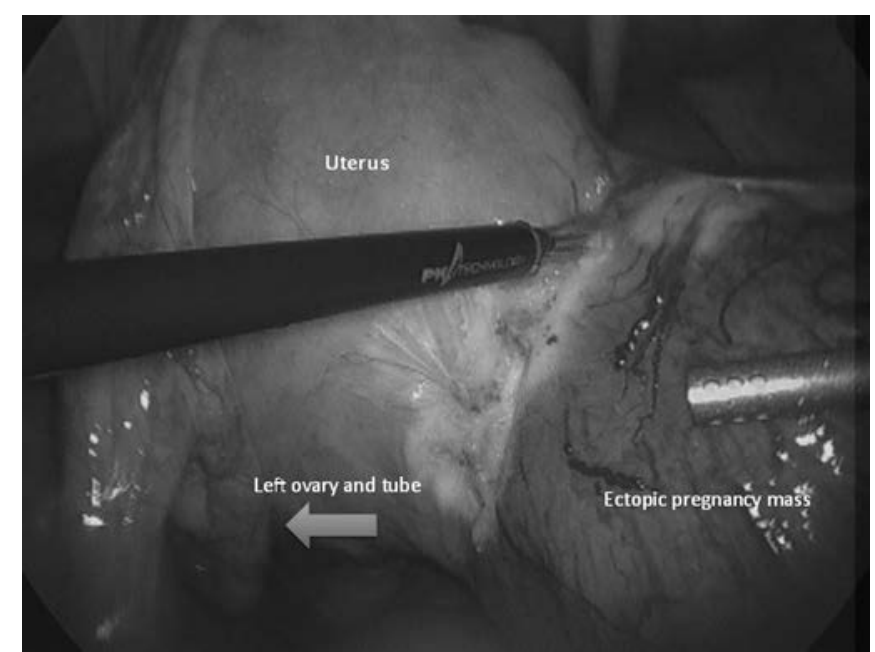

Figure 3. Intraoperative laparoscopic views of systematic circumferential coagulation of the mass from the uterus.

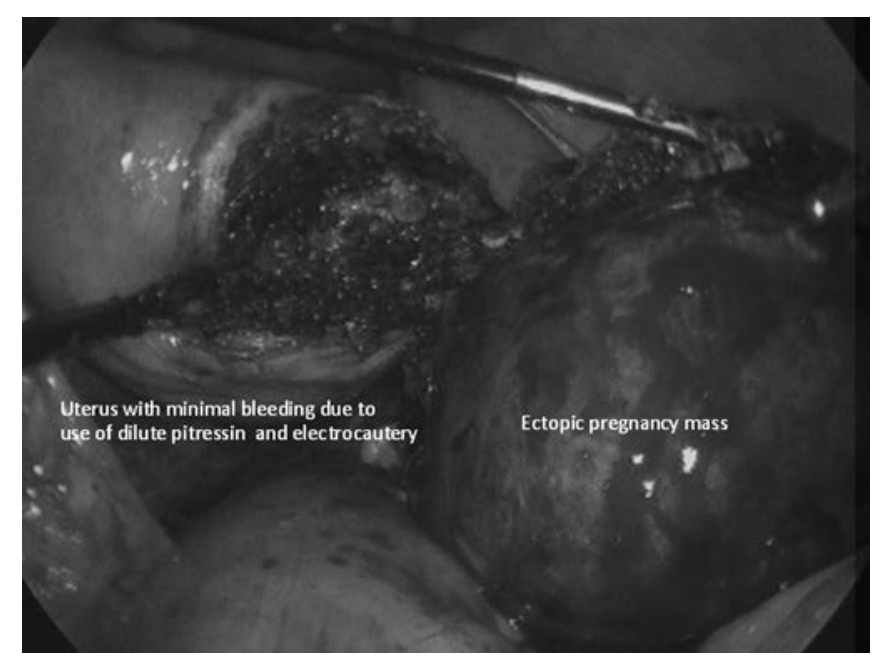

Figure 4. The interstitial pregnancy transected off the uterus. Note the hemostasis at the operative site from both injection of dilute vasopressin and careful use of electrocautery.

The umbilical incision was then extended by $1 \mathrm{~cm}$, and the specimen was removed from the abdominal cavity with a 15-mm Endo Catch device (Covidien) with extracorporeal morcellation required because of a mass size of approximately 8 to $10 \mathrm{~cm}$. All fetal parts were accounted for. The operative time was approximately 90 minutes.

\section{Postoperative Course}

The patient was admitted overnight for observation and was discharged home the following day. The BHCG level was checked on postoperative day 1 and noted to 17,327 $\mathrm{mIU} / \mathrm{mL}$. The patient had an uneventful postoperative 


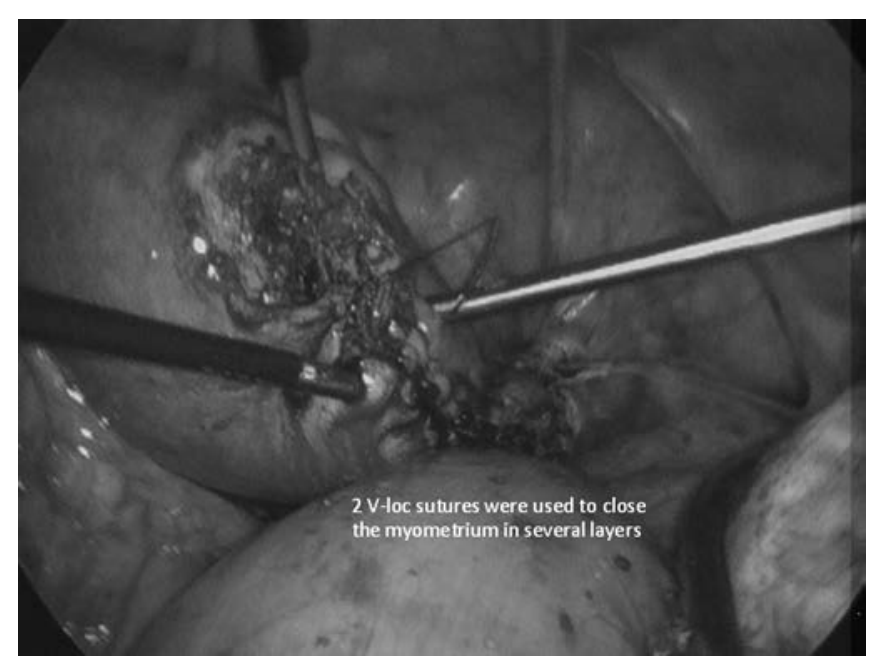

Figure 5. Intraoperative laparoscopic view of the reapproximation of the uterine myometrium in several layers via laparoscopic suturing.

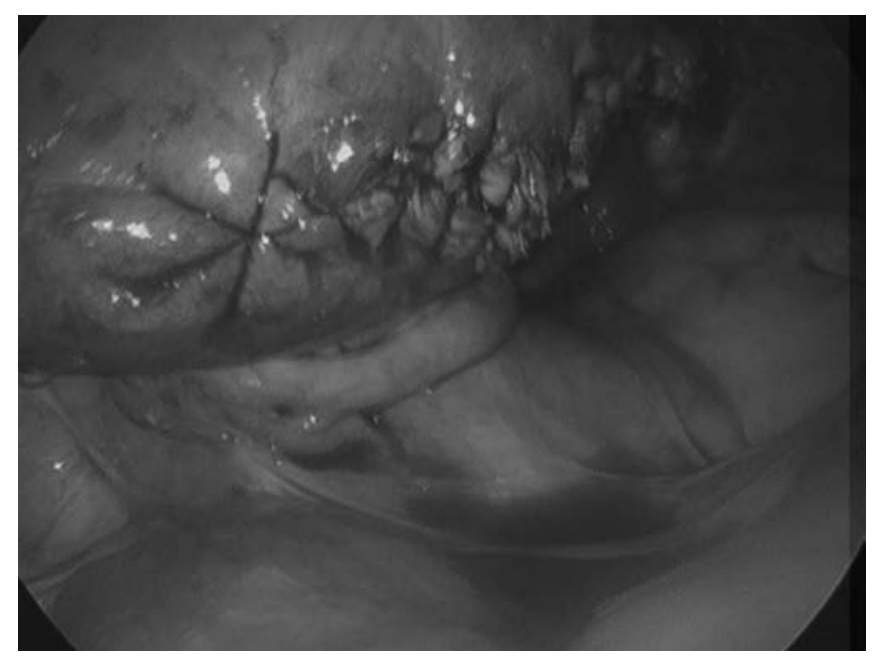

Figure 6. Intraoperative laparoscopic view of uterus after cornual wedge resection and laparoscopic closure. Note hemostasis and swell approximated uterine tissue.

course and has recovered fully. The BHCG level was checked 1 month after the procedure and noted to be 42 $\mathrm{mIU} / \mathrm{mL}$; the patient was subsequently lost to follow-up.

\section{DISCUSSION}

Laparoscopic management of interstitial ectopic pregnancies has been reported extensively in the literature. The mean gestational age in the published case series and reports is 8 weeks, ${ }^{3,16}$ with a mean mass diameter of 3.5 cm. ${ }^{16}$ The largest reported mass that was successfully resected laparoscopically was $6 \mathrm{~cm} .{ }^{16}$ Our case demon- strates that in experienced hands, the laparoscopic management of interstitial ectopic pregnancies up to 13 weeks' gestation and a mass diameter of $8 \mathrm{~cm}$ is a feasible option.

We used 3 techniques to minimize blood loss and complete the surgery laparoscopically: injection of dilute vasopressin both into the cervical stroma and around the base of the pregnancy mass, electrocautery, and multilayer closure of the remaining myometrium and serosa. Multilayer closure will minimize postoperative myometrial bleeding and maximize myometrial thickness and strength, reducing the risk for future uterine rupture. The use of extracorporeal morcellation of the specimen in a laparoscopic bag for the successful removal of the interstitial pregnancy is an important final step to complete a minimally invasive procedure and prevent any spread of products of conception.

The patient had an uneventful and easy recovery. Her BHCG level dropped exponentially over 1 month; however, the patient did not follow-up as recommended until the level was undetectable. Moawad et $\mathrm{al}^{4}$ suggested following BHCG levels to resolution after successful surgical resection to ensure that no persistent interstitial pregnancy tissue remains.

This case illustrates that careful application of minimally invasive techniques for complex gynecologic conditions by experienced surgeons will continue to benefit both individual patients and the health care system.

\section{References:}

1. Ectopic pregnancy-United States 1990-1992. MMWR Morbid Mortal Wkly Rep. 1995;44:46-48.

2. Van Den Eeden SK, Shan J, Bruce C, Glasser M. Ectopic pregnancy rate and treatment utilization in a large managed care organization. Obstet Gynecol. 2005;105:1052-1057.

3. Warda H, Mamik M, Ashraf M, Abuzeid M. Interstitial ectopic pregnancy: conservative surgical management. JSLS. 2014;18: 197-203.

4. Moawad NS, Mahajan ST, Moniz MH, Taylor SE, Hurd WW. Current diagnosis and treatment of interstitial pregnancy. Obstet Gynecol. 2010;202:15-29.

5. Lau S, Tulandi T. Conservative medical and surgical management of interstitial ectopic pregnancy. Fertil Steril. 1999;72: 207-215.

6. Felmus LB; Pedowitz P. Interstitial pregnancy: a survey of 45 cases. AmJ Obstet Gynecol. 1953;66:1271-1279. 
7. Timor-Tritsch IE, Monteagudo A, Matera C, Veit CR. Sonographic evolution of cornual pregnancies treated without surgery. Obstet Gynecol. 1992;79:1044-1049.

8. Tulandi T, Al-Jaroudi D. Interstitial pregnancy: results generated from the Society of Reproductive Surgeons Registry. $\mathrm{Ob}$ stet Gynecol. 2004;103:47-50.

9. Oron G, Tulandi T. A pragmatic and evidence-based management of ectopic pregnancy. J Minim Invasive Gynecol. 2013; $20: 446-454$.

10. Nieboer TE, Johnson N, Lethaby A, Tavender E, Curr E, Garry R, et al. Surgical approach to hysterectomy for benign gynaecological disease. Cochrane Database Syst Rev. 2009;(3): CD003677.

11. Pasic RP, Hammons G, Gardner JS, Hainer M. Laparoscopic treatment of cornual heterotopic pregnancy. J Am Assoc Gynecol Laparosc. 2002;9(3):372-375.

12. Fritz RB, Rosenblum N, Gaither K, Sherman A, McCalla A. Successful laparoscopically assisted transcervical suction evacuation of interstitial pregnancy following failed methotrexate injection in a community hospital setting. Case Rep Obstet Gynecol. 2014;2014:695293.

13. Cai Z, Wang F, Cao H, Xia Q, Chen X, Cai Y. The value of laparoscopy alone or combined with hysteroscopy in the treatment of interstitial pregnancy: analysis of 22 cases. Arch Gynecol Obstet. 2012;285:727-732.
14. Cucinella G, Rotolo S, Calagna G, Granese R, Agrusa A, Perino A. Laparoscopic management of interstitial pregnancy: the "purse-string" technique. Acta Obstet Gynecol Scand. 2012; 91:996-999.

15. Api M, Api O. Laparoscopic cornuotomy in the management of an advanced interstitial ectopic pregnancy: a case report. Gynecol Endocrinol. 2010;26:208-212.

16. Ng S, Hamontri S, Chua I, Chern B, Siow A. Laparoscopic management of 53 cases of cornual ectopic pregnancy. Fertil Steril 2009;92:448-452.

17. Nezhat C, Dun E. Laparoscopically-assisted, hysteroscopic removal of an interstitial pregnancy with a fertility-preserving technique. J Minim Invasive Gynecol. 2014;21:1091-1094.

18. Parker WH, Einarsson J, Istre O, Dubuisson J. Risk factors for uterine rupture after laparoscopic myomectomy. J Minim Invasive Gynecol. 2010;17:551-554.

19. Cobellis L, Pecori E, Cobellis G. Comparison of intramural myomectomy scar after laparotomy or laparoscopy. Int J Gynaecol Obstet. 2004;84:87-88.

20. Guarniccia MM, Rein MS. Traditional surgical approaches to uterine fibroids: abdominal myomectomy and hysterectomy. Clin Obstet Gynecol. 2001;44:385-400.

21. West S, Ruiz R, Parker W. Abdominal myomectomy in women with very large uterine size. Fertil Steril. 2006;85:36-39. 